\title{
Verification of the Constraint Force Equation Methodology for Modeling Multibody Stage Separation
}

\author{
Paul V. Tartabini, ${ }^{*}$ Carlos Roithmayr ${ }^{\dagger}$ \\ NASA Langley Research Center, Hampton, Virginia 23681-2199 \\ Matthew D. Toniolo, ${ }^{\star}$ Christopher Karlgaard ${ }^{\star}$ \\ Analytical Mechanics Associates Inc., Hampton, Virginia 23681 \\ and \\ Bandu N. Pamadi ${ }^{\S}$ \\ NASA Langley Research Center, Hampton, Virginia 23681-2199
}

\begin{abstract}
This paper discusses the verification of the Constraint Force Equation (CFE) methodology and its implementation in the Program to Optimize Simulated Trajectories II (POST2) for multibody separation problems using three specially designed test cases. The first test case involves two rigid bodies connected by a fixed joint; the second case involves two rigid bodies connected with a universal joint; and the third test case is that of Mach 7 separation of the Hyper-X vehicle. For the first two cases, the POST2/CFE solutions compared well with those obtained using industry standard benchmark codes, namely AUTOLEV and ADAMS. For the Hyper-X case, the POST2/CFE solutions were in reasonable agreement with the flight test data. The CFE implementation in POST2 facilitates the analysis and simulation of stage separation as an integral part of POST2 for seamless end-to-end simulations of launch vehicle trajectories.
\end{abstract}

\section{Nomenclature}

$\begin{array}{ll}A, \mathrm{~B} & =\text { Rigid Body } A \text {, and } B \\ \bar{A}, \bar{B} & =\text { Joint location in Body } A \text {, and } B \\ \mathrm{a}_{\mathrm{x}}, \mathrm{a}_{\mathrm{y}}, \mathrm{a}_{\mathrm{z}} & =\text { Components of sensed mass center acceleration in local body frame } \\ \alpha & =\text { Angle-of-attack } \\ \beta & =\text { Angle-of-sideslip } \\ \Delta \mathrm{x}, \Delta \mathrm{y}, \Delta \mathrm{z} & =\mathrm{x}, \mathrm{y} \text { and z components of joint translational displacement in local body frame } \\ \mathbf{I}_{A}, \mathbf{I}_{B} & =\text { Inertia tensor about mass center for Body } A \text {, and } B \\ \mathbf{F}_{A}^{(\mathrm{CON})}, \mathbf{F}_{B}^{(\mathrm{CON})} & =\text { Joint constraint force vector for Body } A \text {, and } B \\ \mathrm{~F}_{x}^{(\mathrm{CON})}, \mathrm{F}_{y}^{(\mathrm{CON})}, \mathrm{F}_{z}^{(\mathrm{CON})} & \text { Constraint force components in local body frame } \\ \mathbf{F}_{A}^{(\mathrm{EXT})}, \mathbf{F}_{B}^{(\mathrm{EXT})} & =\text { External force vector acting on Body } A \text {, and } B \\ \mathrm{~F}_{x}^{(\mathrm{EXT})}, \mathrm{F}_{y}^{(\mathrm{EXT})}, \mathrm{F}_{z}^{(\mathrm{EXT})} & =\text { External force components in local body frame } \\ \mathrm{m}_{A}, \mathrm{~m}_{B} & =\text { Mass of Body } A \text {, and } B \\ \boldsymbol{\omega}_{A}, \boldsymbol{\omega}_{B} & =\text { Angular velocity vector of Body A, and } B \text { relative to an inertial reference frame } \\ \mathbf{r}_{A}, \mathbf{r}_{B} & =\text { Inertial position vector of Body } A, \text { and } B \\ \boldsymbol{\rho}_{A}, \boldsymbol{\rho}_{B} & =\text { Vector from Body A mass center to } \bar{A}, \text { and Body } B \text { mass center to } \bar{B} \\ \mathbf{T}_{A}^{(\mathrm{CON})}, \mathbf{T}_{B}^{(\mathrm{CON})} & =\text { Joint constraint torque vector for Body } A \text {, and } B\end{array}$

\footnotetext{
*Aerospace Engineer, Vehicle Analysis Branch, Member AIAA.

${ }^{\dagger}$ Aerospace Engineer, Space Mission Analysis Branch, Senior Member AIAA.

* Aerospace Engineer, Member AIAA.

$\S$ Aerospace Engineer, Associate Fellow AIAA.
} 


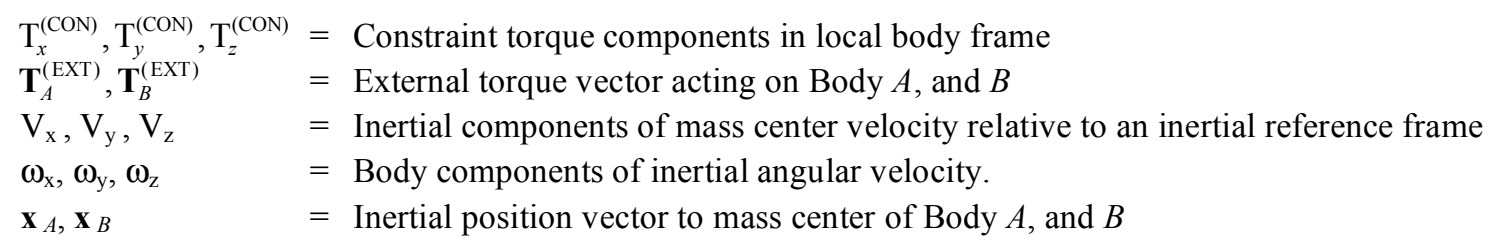

\section{Introduction}

Analyzing the dynamic separation of multiple bodies within the atmosphere is complex and challenging. One problem that has received significant attention in the literature is that of store separation from aircraft. ${ }^{1}$ A similar example is the separation of the X-15 research vehicle from the B-52 carrier aircraft. ${ }^{2}$ In both of these cases, the store and the X-15 vehicle are much smaller in size than the parent vehicle. The other class of stage separation problem involves separation of two vehicles of comparable sizes, as in the case of multi-stage reusable launch vehicles where the integrity of each stage is important after separation.

NASA studies on stage separation of multi-stage reusable launch vehicles date back to the early $1960 \mathrm{~s} \cdot{ }^{3-7}$ These studies addressed the problem of separation of generic two-stage reusable launch vehicles. More recently, Naftel et al. considered staging of dual wing-body vehicles. ${ }^{8-10}$ NASA's interest in stage separation research was renewed in early 2000 when it was realized that the technologies needed for the development of a next generation, reusable single-stage-to-orbit vehicle were not yet available and the focus shifted to multi-stage launch vehicles. Accordingly, NASA initiated a comprehensive stage separation tool development activity that included wind tunnel testing as well as development and validation of CFD and engineering level simulation tools. ${ }^{11}$ As a part of this activity, a stage separation analysis and simulation tool was developed called ConSep (short for Conceptual Separation), which is a front end to the commercial multibody dynamic analysis software ADAMS ${ }^{\circledR} .{ }^{12}$ References 13-15 discuss ConSep, and its application to two-body and three-body separation problems. One disadvantage of ConSep is that it cannot be easily integrated into standard trajectory simulation software for performing efficient and seamless end-to-end simulations of launch vehicle trajectories because it is tied to ADAMS. To address this problem, the authors developed the CFE methodology and implemented it into the Program to Simulate Optimized Trajectories II (POST2). ${ }^{16}$

The objective of this paper is to test and verify the CFE methodology, presented by the authors in Ref. 16, as a means in which any standard trajectory simulation program capable of simulating multiple unconnected vehicles can be modified, with a minimal set of changes, to solve constrained motion problems; that is, problems where vehicles are connected by simple joints. Although the CFE methodology is generic in nature and can be implemented in any conventional trajectory simulation program, this paper discusses its implementation in the industry standard trajectory simulation and optimization software package, POST2. ${ }^{17,18}$

By itself, POST2 does not have the capability to model internal joint forces and moments prior to separation when the bodies are still connected. The CFE implementation in POST2 provides a framework for computing the internal constraint forces and moments acting at joints connecting multiple vehicles and applies them as external forces/moments on each body, along with the usual external forces and moments due to gravity, aerodynamics, and propulsion that are computed by POST2. Thus, the CFE methodology simply augments the external vehicle external loads and does not require modification of the POST2 equations of motion. It provides the missing link to accurately model the dynamics of generic stage separation problems.

In this paper the CFE methodology is briefly reviewed, and some of the steps taken to verify and validate its results are highlighted. In particular, its application to three test cases of increasing complexity is described. The first test case simulates the motion of two rigid bodies connected by a fixed joint with no external forces. The advantage of studying this basic problem is that numerical results can be compared with what is to be expected on the basis of first principles. The next test case involves comparisons between results obtained with CFE and ADAMS for two rigid bodies connected by a universal joint. Finally, the paper concludes with a comparison of a POST2/CFE simulation of the Mach 7 separation of the X-43A vehicle to previously published POST2 results (generated using an engineering model) and the flight data. These three test cases were part of a larger, comprehensive series of checks that were performed on the CFE technique that provide confidence in its ability to solve constrained motion problems in conventional trajectory programs like POST2. For the first two cases, the CFE simulations are in excellent agreement with simulations using benchmark, industry standard software. For the third test case, CFE performs as well as the engineering model but is easier to implement, and the results compare reasonably well with the flight data. 


\section{Constraint Force Equation Methodology}

To illustrate the basic concept of the CFE methodology, consider the motion of two rigid bodies connected by a single joint as shown in Fig. 1. The external forces and moments that act on each body are shown in Fig. 1(a). These external forces $\left(\mathbf{F}_{A}^{(\mathrm{EXT})}\right.$ and $\left.\mathbf{F}_{B}^{(\mathrm{EXT})}\right)$ and external moments or torques about mass centers $\left(\mathbf{T}_{A}^{(\mathrm{EXT})}\right.$ and $\mathbf{T}_{A}^{(\mathrm{EXT})}$ ) are the resultants of gravity, aerodynamic, and propulsive forces on each vehicle and are readily computed by POST2. Figure 1(b) shows the internal constraint forces and moments $\left(\mathbf{F}^{(\mathbf{C O N})}\right.$ and $\left.\mathbf{T}^{(\mathbf{C O N})}\right)$ computed by CFE that act on each vehicle at the joint loca-

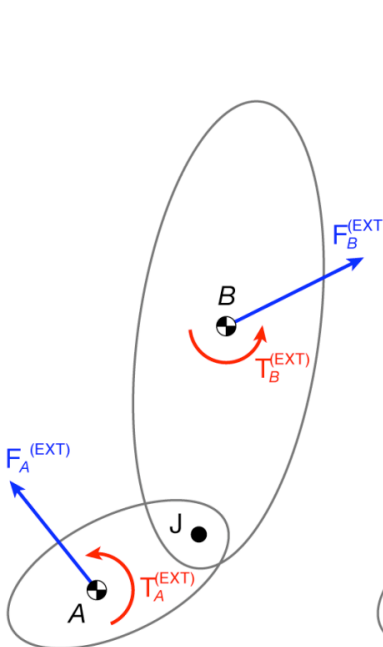

a) External forces.

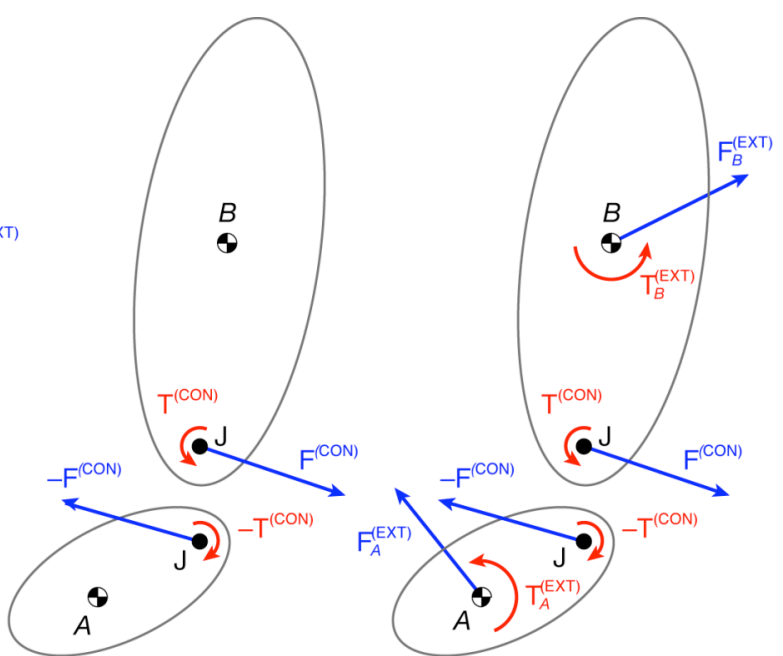

b) Internal forces.

c) Free body diagram. tion. These internal forces and

Figure 1. Decomposition of external forces and internal constraint forces to free body diagram solved by CFE method.

moments constrain the way in which one vehicle can move relative to the other, and are dependent upon the external forces acting on each vehicle as well as the type of joint. For joints that permit only relative rotation between two bodies, the forces and moments on one body have magnitudes that are equal and directions that are opposite to those acting on the other body, as shown in Fig. 1(b). Finally, Fig. 1(c) illustrates the way in which CFE is implemented in POST2. At each integration time step the current version of POST2 computes the typical external forces and moments acting on each vehicle. This information, along with specific geometric information about the joint, is provided to the CFE routine, which works in parallel to compute the internal constraint forces and moments required to satisfy the constraints imposed by the joint. Next, these internal joint loads are applied to each vehicle as additional external forces and moments, and the POST2 solution is propagated in the usual manner to the next time step. Thus, the net external forces and moments on each vehicle are the sum of the usual external forces and moments and the joint loads, applied to each vehicle as additional external forces and moments. Consequently, the CFE joint model simply augments the vehicle external loads and does not require modification to the POST2 equations of motion.

The equations of constrained motion of two rigid bodies $A$ and $B$ connected by a single joint are as follows. For body $A$,

$$
\begin{gathered}
\mathbf{F}_{A}^{(\mathrm{EXT})}+\mathbf{F}_{A}^{(\mathrm{CON})}=m_{A} \ddot{\mathbf{x}}_{A} \\
\mathbf{T}_{A}^{(\mathrm{EXT})}+\boldsymbol{\rho}_{A} \times \mathbf{F}_{A}^{(\mathrm{CON})}+\mathbf{T}_{A}^{(\mathrm{CON})}=\mathbf{I}_{A} \cdot \dot{\boldsymbol{\omega}}_{A}+\boldsymbol{\omega}_{A} \times \mathbf{I}_{A} \cdot \boldsymbol{\omega}_{A}
\end{gathered}
$$

where $\rho_{A}$ is the position vector from the mass center of $A$ to the point $\bar{A}$ of $A$ at which the constraint force is applied. Similarly, for body $B$,

$$
\begin{gathered}
\mathbf{F}_{B}^{(\mathrm{EXT})}+\mathbf{F}_{B}^{(\mathrm{CON})}=m_{B} \ddot{\mathbf{x}}_{B} \\
\mathbf{T}_{B}^{(\mathrm{EXT})}+\boldsymbol{\rho}_{B} \times \mathbf{F}_{B}^{(\mathrm{CON})}+\mathbf{T}_{B}^{(\mathrm{CON})}=\mathbf{I}_{B} \cdot \dot{\boldsymbol{\omega}}_{B}+\boldsymbol{\omega}_{B} \times \mathbf{I}_{B} \cdot \boldsymbol{\omega}_{B}
\end{gathered}
$$

Note that in the case of unconstrained motion, the constraint forces and moments vanish; Eqs. (1)-(4) reduce to the standard equations of motion for two independent rigid bodies. Each mass center acceleration, rigid body angular acceleration, constraint force, and constraint torque are regarded as unknowns; therefore, Eqs. (1)-(4) as they are 
written represent 12 scalar equations in 24 unknowns. Hence, twelve additional scalar equations are required. Of these, six are obtained from two vector relationships that are consequences of the law of action and reaction:

$$
\begin{gathered}
\mathbf{F}_{A}^{(\mathrm{CON})}+\mathbf{F}_{B}^{(\mathrm{CON})}=\mathbf{0} \\
\mathbf{T}_{A}^{(\mathrm{CON})}+\mathbf{T}_{B}^{(\mathrm{CON})}+\left(\mathbf{r}_{B}-\mathbf{r}_{A}\right) \times \mathbf{F}_{B}^{(\mathrm{CON})}=\mathbf{0}
\end{gathered}
$$

where $\mathbf{r}_{A}$ is the position vector from a point fixed in an inertial reference frame to $\bar{A}$. Because $\mathbf{x}_{A}$ is the position vector from a point fixed in an inertial reference frame to the mass center of $A$, we can write $\mathbf{r}_{A}=\mathbf{x}_{A}+\boldsymbol{\rho}_{A}$. It should be kept in mind that the vector $\rho_{A}$ is fixed in $A$. The position vector $\mathrm{r}_{B}$ is defined similarly. Note that the cross product term involving $\left(\mathbf{r}_{B}-\mathbf{r}_{A}\right)$ in Eq. (6) is required for joints that allow translation (see Ref. 16); the equation gives a simple, expected result when no translation is permitted $\left(\mathbf{r}_{B}=\mathbf{r}_{A}\right)$.

Equations (1)-(6) are applicable to each of the three test cases discussed in this paper. The six remaining equations fall into one of two categories. First, equations of constraint are formed that describe the ways in which a particular joint restricts relative translation or relative rotation. Second, by considering the translation or rotation that $i s$ permitted by the joint, equations are written to account for the absence of constraint force or torque in a particular direction in view of the ideal (perfectly smooth) nature of the joint.

When relative translation is constrained, the distance between two points must remain fixed in a particular direction. That is,

$$
\left(\mathbf{r}_{B}-\mathbf{r}_{A}\right) \cdot \mathbf{e}_{A}=0
$$

where $\mathbf{e}_{A}$ is a unit vector fixed in body $A$ in the direction that the translation motion is not permitted by the joint. One equation having the form of Eq. (7) is needed to account for each direction that translation is constrained. For example, a fixed joint constrains translation in three orthogonal directions; therefore, three equations in the form of Eq. (7) are required. In each such equation, the role of $\mathbf{e}_{A}$ is played by one of the three mutually orthogonal unit vectors fixed in $A$. For a prismatic (sliding) joint that permits translation in only one direction (and restricts translation in two perpendicular directions), two equations having the form of Eq. (7) are required.

A constraint on relative rotation can be viewed as a requirement that two unit vectors must remain perpendicular; each unit vector is fixed in one of the bodies and is normal to the axis about which rotation would take place if the constraint were not present. The constraint is expressed by setting the scalar product of the two unit vectors equal to zero.

$$
\mathbf{e}_{B} \cdot \mathbf{e}_{A}=0
$$

where the two unit vectors $\mathbf{e}_{A}$ and $\mathbf{e}_{B}$ (one fixed in each body) are chosen to be perpendicular to each other throughout the constrained motion. One equation in the form of Eq. (8) is required for each direction about which rotation is constrained.

The constraint equations (7) and (8) are differentiated twice with respect to time so that the resulting equations involve the unknown linear and angular accelerations of bodies $A$ and $B$,

$$
\begin{gathered}
\frac{d^{2}}{d t^{2}}\left[\left(\mathbf{r}_{B}-\mathbf{r}_{A}\right) \cdot \mathbf{e}_{A}=0\right] \\
\frac{d^{2}}{d t^{2}}\left[\mathbf{e}_{B} \cdot \mathbf{e}_{A}=0\right]
\end{gathered}
$$

The result of differentiating Eq. (7) once is

$$
\left(\dot{\mathbf{r}}_{B}-\dot{\mathbf{r}}_{A}\right) \cdot \mathbf{e}_{A}+\left(\mathbf{r}_{B}-\mathbf{r}_{A}\right) \cdot\left(\boldsymbol{\omega}_{A} \times \mathbf{e}_{A}\right)=0
$$


where $\dot{\mathbf{r}}_{A}$ and $\dot{\mathbf{r}}_{B}$ are, respectively, the time derivatives in an inertial reference frame of $\mathbf{r}_{A}$ and $\mathbf{r}_{B}$. Differentiating once more, one obtains

$$
\begin{aligned}
& \left(\ddot{\mathbf{x}}_{B}+\dot{\boldsymbol{\omega}}_{B} \times \boldsymbol{\rho}_{B}-\ddot{\mathbf{x}}_{A}-\dot{\boldsymbol{\omega}}_{A} \times \boldsymbol{\rho}_{A}\right) \cdot \mathbf{e}_{A}+\left(\mathbf{r}_{B}-\mathbf{r}_{A}\right) \cdot\left(\dot{\boldsymbol{\omega}}_{A} \times \mathbf{e}_{A}\right)= \\
& 2\left(\dot{\mathbf{r}}_{B}-\dot{\mathbf{r}}_{A}\right) \cdot\left(\mathbf{e}_{A} \times \boldsymbol{\omega}_{A}\right)-\left(\mathbf{r}_{B}-\mathbf{r}_{A}\right) \cdot\left(\boldsymbol{\omega}_{A} \times \boldsymbol{\omega}_{A} \times \mathbf{e}_{A}\right)+\left[\left(\boldsymbol{\omega}_{A} \times \boldsymbol{\omega}_{A} \times \boldsymbol{\rho}_{A}\right)-\left(\boldsymbol{\omega}_{B} \times \boldsymbol{\omega}_{B} \times \boldsymbol{\rho}_{B}\right)\right] \cdot \mathbf{e}_{A}
\end{aligned}
$$

Differentiating once with respect to time in Eq. (8) yields

$$
\left(\boldsymbol{\omega}_{B} \times \mathbf{e}_{B}\right) \cdot \mathbf{e}_{A}+\mathbf{e}_{B} \cdot\left(\boldsymbol{\omega}_{A} \times \mathbf{e}_{A}\right)=0
$$

and differentiating once more gives

$$
\left(\dot{\boldsymbol{\omega}}_{B}-\dot{\boldsymbol{\omega}}_{A}\right) \cdot\left(\mathbf{e}_{B} \times \mathbf{e}_{A}\right)=\left(\boldsymbol{\omega}_{B}-\boldsymbol{\omega}_{A}\right) \cdot\left[\mathbf{e}_{A} \times\left(\boldsymbol{\omega}_{B} \times \mathbf{e}_{B}\right)-\mathbf{e}_{B} \times\left(\boldsymbol{\omega}_{A} \times \mathbf{e}_{A}\right)\right]
$$

When relative translation is permitted in a certain direction, an equation having the form of Eq. (12) is not required. Likewise, when relative rotation is allowed, a relationship in the form of Eq. (14) is not necessary. Instead, the required equations can be obtained using the condition that the constraint forces or moments are zero in those directions (with the assumption that the surfaces of the joint are perfectly smooth). Thus, for a joint that permits translation in a certain direction,

$$
\mathbf{F}^{(\mathrm{CON})} \cdot \mathbf{e}=0
$$

Depending on the circumstances, $\mathbf{F}^{(\mathrm{CON})}$ can be the constraint force acting either on $A$ or on $B$. Similarly, when a joint permits relative rotation in a certain direction,

$$
\mathbf{T}^{(\mathrm{CON})} \cdot \mathbf{e}=0
$$

In summary, equations having the form of Eqs. (12) and (14)-(16) provide, in combination, a total of six scalar equations.

The 24 scalar equations formed from Eqs. (1)-(6), (12), and (14)-(16) are linear in 24 unknowns; 3 variables represent each mass center acceleration, rigid body angular acceleration, constraint force, and constraint moment for each of the two rigid bodies. These equations can be expressed in matrix form, $A x=b$ (where the column matrix $x$ contains the 24 unknown parameters) and solved accordingly using a standard matrix inversion technique. The matrix A can be singular when, for example, one neglects a central principal moment of inertia, such as for a slender rod, or when the mass of a body is neglected. The matrix also becomes singular when constraint equations are redundant; in other words, when one is linearly dependent on the others.

\section{Test Cases}

A suite of test cases was developed to check and verify the CFE methodology for application to multibody separation problems in the launch vehicle staging environment. These test cases are listed in Table 1 and cover the types of joints typically needed to model stage separation problems. The list is not exhaustive, and, in general, it is possible to model

Table 1. Test Cases Performed for CFE Verification

\begin{tabular}{lcc}
\hline \multicolumn{1}{c}{ Joint Type } & $\begin{array}{c}\text { Translational Degrees } \\
\text { of Freedom }\end{array}$ & $\begin{array}{c}\text { Rotational Degrees } \\
\text { of Freedom }\end{array}$ \\
\hline Fixed & 0 & 0 \\
Revolute (Hinge) & 0 & 1 \\
Universal & 0 & 2 \\
Ball & 0 & 3 \\
1-D Translational (Slider) & 1 & 0 \\
2-D Translational (Planar) & 2 & 0 \\
Cylindrical & 1 & 1 \\
\hline \hline
\end{tabular}


joints that simultaneously constrain any combination of translational and rotational degrees of freedom with CFE simply by forming the appropriate equations described in Eqs. (12) and (14)-(16).

Two test cases from Table 1 (the fixed joint and the universal joint) are described in this paper to illustrate the testing and verification that was performed on the CFE methodology. A third test case was conducted that utilized POST2/CFE to model the Hyper-X stage separation problem as a 1-D translational (or sliding) joint in series with a revolute (or hinge) joint.

\section{A. Test Case 1: Fixed Joint}

This test case involves two rigid bodies, denoted as bodies $A$ and $B$, connected by a fixed joint (see Fig. 2). The mass properties for each body are shown in Table 2 . The fixed joint constrains a point $\bar{A}$ fixed in $A$ to remain coincident with a point $\bar{B}$ fixed in $B$. Thus, three constraint equations having the form of Eq. (7) can be written as

$$
\left(\mathbf{r}_{B}-\mathbf{r}_{A}\right) \cdot \hat{\mathbf{a}}_{r}=0 \quad(r=1,2,3)
$$

where the role of $\mathbf{e}_{A}$ in Eq. (7) is played in turn by $\hat{\mathbf{a}}_{1}, \hat{\mathbf{a}}_{2}$, and $\hat{\mathbf{a}}_{3}$, each of which belongs to a set of three righthanded, mutually orthogonal unit vectors fixed in $A$ as shown in Fig. 2 . Suppose that $\hat{b}_{1}, \hat{b}_{2}$, and $\hat{b}_{3}$ are a similar set

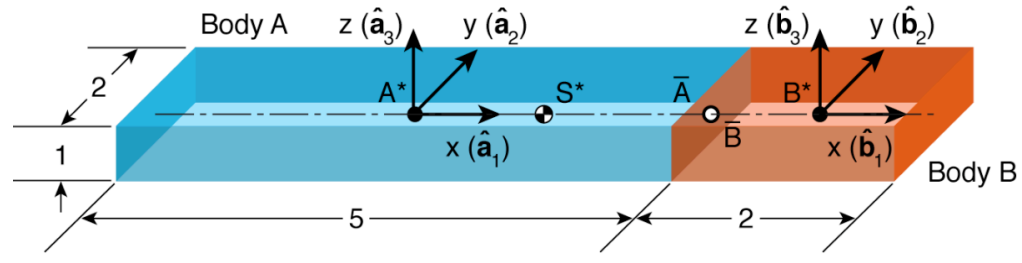

Figure 2. Test Case 1: Two rigid bodies, $A$ and $B$, connected by a fixed joint.
Table 2. Mass Properties for Test Case 1, Fixed Joint

\begin{tabular}{ccc}
\hline \hline & Body $A$ & Body $B$ \\
\hline$m$ & $1.0 \mathrm{~kg}$ & $0.4 \mathrm{~kg}$ \\
$I_{x x}$ & $0.42 \mathrm{~kg}-\mathrm{m}^{2}$ & $0.17 \mathrm{~kg}-\mathrm{m}^{2}$ \\
$I_{y y}$ & $2.17 \mathrm{~kg}-\mathrm{m}^{2}$ & $0.17 \mathrm{~kg}-\mathrm{m}^{2}$ \\
$I_{z z}$ & $2.42 \mathrm{~kg}-\mathrm{m}^{2}$ & $0.27 \mathrm{~kg}-\mathrm{m}^{2}$ \\
\hline \hline
\end{tabular}

of unit vectors fixed in $B$ such that $\hat{\mathbf{b}}_{r}$ has the same direction as $\hat{\mathbf{a}}_{r}(r=1,2,3)$ when $A$ and $B$ are attached to each other. In that case, the fixed joint constrains certain unit vectors fixed in $A$ to remain perpendicular to other unit vectors fixed in $B$. For example, one can write the following three constraint equations having the form of Eq. (8).

$$
\begin{aligned}
& \hat{\mathbf{a}}_{1} \cdot \hat{\mathbf{b}}_{2}=0 \\
& \hat{\mathbf{a}}_{1} \cdot \hat{\mathbf{b}}_{3}=0 \\
& \hat{\mathbf{a}}_{3} \cdot \hat{\mathbf{b}}_{2}=0
\end{aligned}
$$

Thus, a total of six constraint equations have been written. As mentioned previously, these constraint equations are differentiated twice with respect to time so that the resulting relationships involve the unknown accelerations and angular accelerations. Thus, one employs three equations having the form of Eq. (12) in which the role of $\mathbf{e}_{A}$ is played by $\hat{\mathbf{a}}_{1}, \hat{\mathbf{a}}_{2}$, and $\hat{\mathbf{a}}_{3}$ respectively. Likewise, three equations having the form of Eq. (14) come into play. In the first of these, the roles of $\mathbf{e}_{A}$ and $\mathbf{e}_{B}$ are played by $\hat{\mathbf{a}}_{1}$ and $\hat{\mathbf{b}}_{2}$. In the second such equation, $\hat{\mathbf{a}}_{1}$ and $\hat{\mathbf{b}}_{3}$ play the parts of $\mathbf{e}_{A}$ and $\mathbf{e}_{B}$. In the third relationship, $\hat{\mathbf{a}}_{3}$ and $\hat{\mathbf{b}}_{2}$ are substituted for $\mathbf{e}_{A}$ and $\mathbf{e}_{B}$.

No relationships having the form of Eqs. (15) or (16) are applicable in the case of the fixed joint because no relative motion is permitted by the joint.

\section{B. Test Case 2: Universal Joint}

This test case involves two rigid bodies, $A$ and $B$, connected by a universal joint. This configuration is shown in Fig. 3. The universal joint constrains a point $\bar{B}$ fixed in $B$ to remain coincident with a point $\bar{A}$ fixed in $A$, at the intersection of the two arms in the cross. Therefore, the three translational constraint equations presented in Eq. (17) for the fixed joint apply in this case as well. 
For this problem, one additional rotational constraint equation having the form of Eq. (8) is needed. If $\hat{\mathbf{a}}_{2}$ is chosen to be parallel to the arm that is fixed in $A$, and $\hat{\mathbf{b}}_{3}$ is chosen to be parallel to the arm that is fixed in $B$, then the rotational constraint equation imposed by the universal joint can be described with the relationship

$$
\hat{\mathbf{b}}_{3} \cdot \hat{\mathbf{a}}_{2}=0
$$

The foregoing four constraint equations are differentiated twice with respect to time. Thus, for the translational constraint one employs three equations having the form of Eq. (12) in which the role of $\mathbf{e}_{A}$ is played by $\hat{\mathbf{a}}_{1}$, $\hat{\mathbf{a}}_{2}$, and $\hat{\mathbf{a}}_{3}$ respectively. Likewise, for the rotational constraint the result is obtained by employing Eq. (14) and making two substitutions: $\hat{\mathbf{a}}_{2}$ for $\hat{\mathbf{e}}_{A}$, and $\hat{\mathbf{b}}_{\mathbf{3}}$ for $\hat{\mathbf{e}}_{B}$.

The other two equations are obtained with the aid of Eq. (16): it is assumed that the universal joint is ideal (frictionless), so that no constraint torque is exerted in the directions of

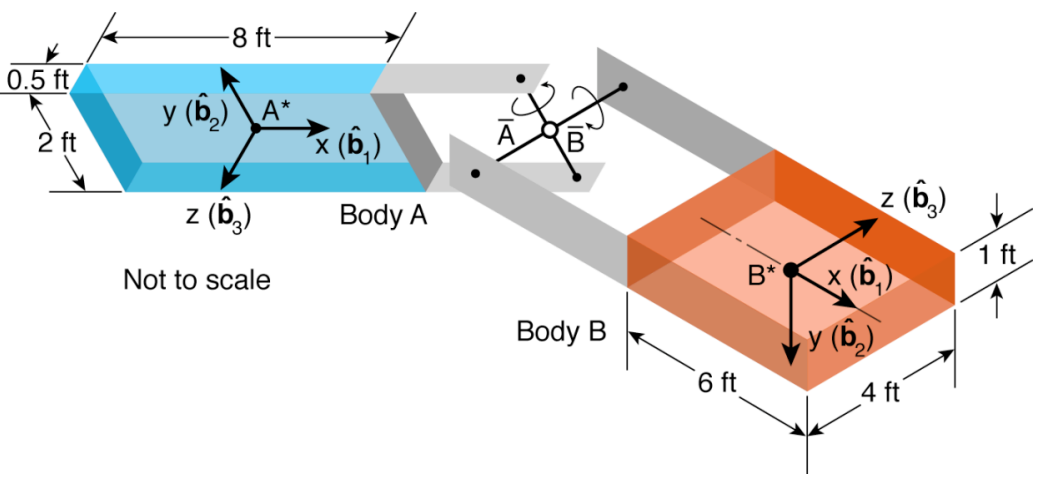

Figure 3. Test Case 2: Two rigid bodies, $A$ and $B$, connected by a universal joint.

the arms in the cross.

$$
\mathbf{T}_{A}{ }^{(\mathrm{CON})} \cdot \hat{\mathbf{a}}_{2}=0, \quad \mathbf{T}_{B}{ }^{(\mathrm{CON})} \cdot \hat{\mathbf{b}}_{3}=0
$$

The mass properties of each vehicle are summarized in Table 3 (products of inertia are zero for each body in this problem).

\section{Test Case 3: Hyper X}

This test case was selected to evaluate the capability of POST2/CFE to model and simulate a realistic stage separation problem by comparing results with flight data from NASA's X-43A hypersonic scramjet test vehicle (see Fig. 4). In 2004, NASA conducted two successful

Table 3. Mass properties for Test Case 2, Universal Joint

\begin{tabular}{ccc}
\hline & Body $A$ & Body $B$ \\
\hline$m$ & 622.0 slugs & 622.0 slugs \\
$I_{x x}$ & 220.3 slug- $\mathrm{ft}^{2}$ & 881.2 slug- $\mathrm{ft}^{2}$ \\
$I_{y y}$ & 3524.7 slug- $\mathrm{ft}^{2}$ & 2695.3 slug- $\mathrm{ft}^{2}$ \\
$I_{z z}$ & 3330.3 slug- $\mathrm{ft}^{2}$ & 1917.8 slug- $\mathrm{ft}^{2}$ \\
\hline \hline
\end{tabular}
scramjet test flights of the X-43A research vehicle (RV) at speeds near Mach 7 and 10, respectively. The roughly 10 second scramjet test was performed after the RV was boosted to the target flight condition by the Hyper-X Launch Vehicle (HXLV). A dimensioned drawing of the mated RV and HXLV is shown in Fig. 5. Additional information describing the Hyper-X RV and HXLV may be found in Reference 19.

The X-43A stage separation event was initiated approximately three seconds after HXLV burnout at a dynamic pressure of $1000 \mathrm{psf}$. Separation began when two pyrotechnically actuated pistons extending from the booster pushed against the RV to induce $\sim 16 \mathrm{ft} / \mathrm{s}$ of relative velocity between the two vehicles. The two pistons, which were positioned roughly 9 in. on each side of the RV centerline, were initially in contact with a cup-like ball joint attached to the RV that permitted rotation about the piston contact point and translation only along the line-of-action of the piston force, which was oriented 4 deg below the horizontal. Both pistons were connected to the same gas chamber and were activated at the same time and remained in contact with the $\mathrm{RV}$ for $\sim 0.1 \mathrm{sec}$ until they reached the end of their 9-in. stroke length. Although the ball joint permitted rotation in any direction at each individual piston contact point, the fact

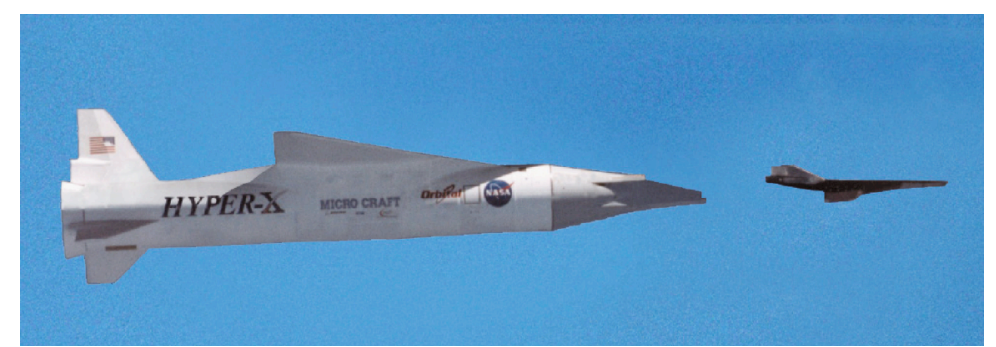

Figure 4. Test Case 3. Artistic rendering of X-43A (RV) separation from HXLV booster.

American Institute of Aeronautics and Astronautics 
that both pistons were fired concurrently and were simultaneously in contact with the RV effectively excluded relative rolling and yawing rotation between the two separating vehicles.

Once the pistons reached the end of their 9-inch extensions, the RV separated from the HXLV. At this time, the HXLV commanded a nose-down pitching moment to move away from the RV, and the RV was steered to its required target condition.

To test the CFE methodology, this problem was set up using POST2/CFE to model the piston constraint forces. For the purpose of this paper, the two pistons were modeled as a single piston to avoid over-constraining the problem. Thus, a single piston was modeled as a sliding joint in series with a revolute joint; that is, relative translation was allowed in one direction (the piston line-ofaction) and relative rotation was permitted only about the pitch axis. The piston was placed at the centerline of the RV and the axial force that pushed the vehicles apart was doubled. A schematic highlighting these modeling details is shown in Fig. 6.

To illustrate how the constraint equations for this joint are derived, unit vectors are defined in the RV such that $\hat{\mathbf{a}}_{1}$ is the direction in which translation or sliding is permitted (along the piston line-of-action) and $\hat{\mathbf{a}}_{2}$ indicates the direction in which rotation is free to occur (pitch axis). A similar set of unit vectors, $\hat{\mathbf{b}}_{r}$, can be defined that are fixed in the HXLV and initially aligned with the $\hat{\mathbf{a}}_{r}$ unit vectors fixed in the RV (see Fig. 6). For this case, two constraint equations having the form of Eq. (7) can be written as:

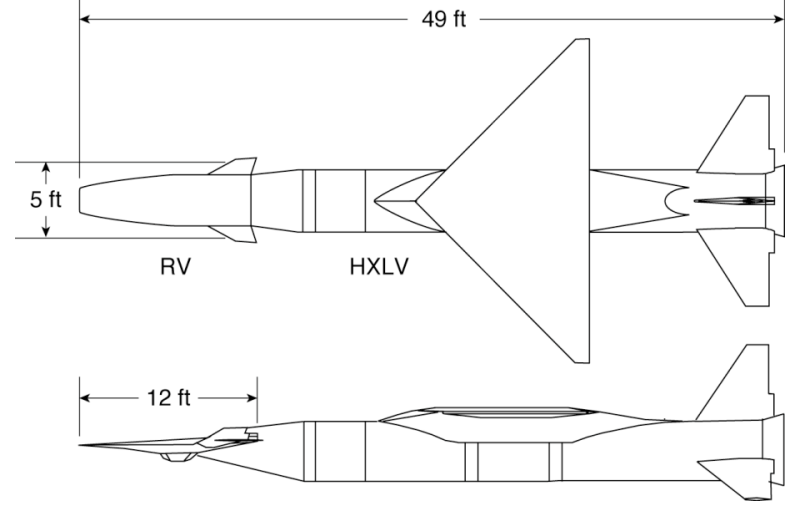

Figure 5. Test Case 3: Dimensioned drawing of mated X-43A Research Vehicle and Hyper-X Launch Vehicle.

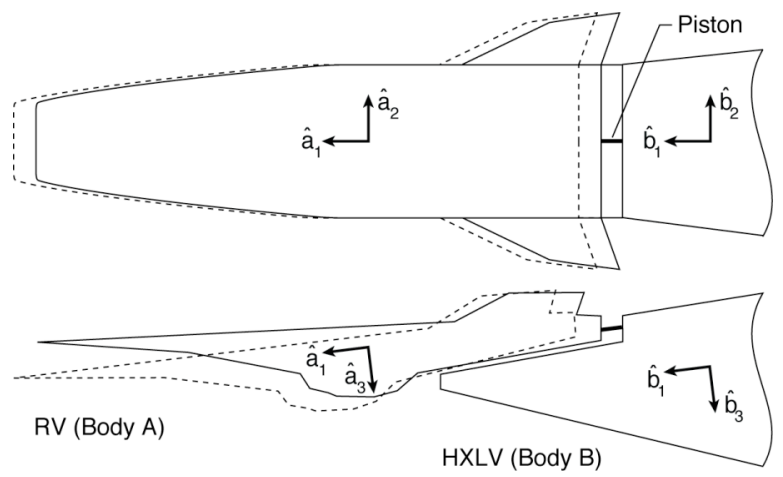

Figure 6. Test Case 3. CFE modeling of piston contact for Hyper-X (X-43A) separation problem.

$$
\left.\left(\mathbf{r}_{B}-\mathbf{r}_{A}\right) \cdot \hat{\mathbf{a}}_{r}=0 \quad(\mathrm{r}=2,3)\right)
$$

As before, these two constraint equations are differentiated twice with respect to time as in Eq. (12). Now, $\hat{\mathbf{a}}_{1}$ and $\hat{\mathbf{a}}_{2}$ are substituted for $\mathbf{e}_{A}$. Furthermore, two constraint equations describing the restriction imposed on relative orientation are formed,

$$
\hat{\mathbf{a}}_{2} \cdot \hat{\mathbf{b}}_{1}=0, \quad \hat{\mathbf{a}}_{2} \cdot \hat{\mathbf{b}}_{3}=0
$$

Similarly, these constraint equations are differentiated twice with respect to time as in Eq. (14), where now $\hat{\mathbf{a}}_{2}$ is substituted for $\mathbf{e}_{A}$, and $\hat{\mathbf{b}}_{1}$ and $\hat{\mathbf{b}}_{3}$ are substituted for $\mathbf{e}_{A}$. With the assumption that the joint is frictionless, the constraint force and moment in the directions where motion is permitted become, using Eqs. (15) and (16),

$$
\mathbf{F}_{B}^{(\mathrm{CON})} \cdot \hat{\mathbf{a}}_{1}=0, \quad \mathbf{T}_{B}^{(\mathrm{CON})} \cdot \hat{\mathbf{a}}_{2}=0
$$




\section{Results and Discussion}

\section{A. Test Case I, Fixed Joint}

The two rigid bodies, $A$ and $B$, are assumed to be rigidly connected to each other for $10 \sec$ (see Fig. 2) and then released instantaneously. No external forces or moments were assumed to act on either body for the entire duration of the 20 -sec simulation. Initial conditions correspond to zero inertial velocity of the system mass center $\left(S^{*}\right)$, and an inertial angular velocity of the rigid body formed by $A$ and $B$ equal to $63.02 \hat{\mathbf{a}}_{1}-82.32 \hat{\mathbf{a}}_{2}+80.25 \hat{\mathbf{a}}_{3} \mathrm{deg} / \mathrm{s}$. After 10 sec, the joint connecting $A$ and $B$ is released, and their motions become unconstrained. Because there are no external forces or moments, integrals of the motion involving linear and angular momentum must remain constant and this fact can be used to check numerical solutions of the equations of motion. Therefore, one can expect the simulation results to show that, after separation, the mass centers of $A$ and $B$ travel in straight lines with constant velocities, whereas the system mass center remains at rest.

To verify the POST2/CFE simulation results, a simulation was also created with AUTOLEV, ${ }^{20-22}$ an interactive program designed specifically for the kinematic and dynamic analysis of mechanical systems. Symbolic manipulation (computer algebra) is used to formulate explicit equations of motion for the particular system of interest using any method; the software is particularly well suited to Kane's method. ${ }^{22-23}$ For this test case, AUTOLEV was used to create a computer program to simulate the motions of $A$ and $B$, and determine the constraint forces required to hold them together for the first 10 seconds.

The AUTOLEV results were generated using a variable step integrator with an absolute error limit of $1 \times 10^{-8}$ and a relative error limit of $1 \times 10^{-7}$. The POST2/CFE method employed a fixed step integrator with a step size of $0.0001 \mathrm{sec}$. The CFE routine also used a Baumgarte ${ }^{24}$ constraint stabilization factor of 5.0.

Time histories for the inertial $x, y$, and $z$ components of velocity of the mass center of body $A$ are shown for 20 $\mathrm{sec}$ in Fig. 7. The values oscillate during the first $10 \mathrm{sec}$, after which the joint is released and their values become constant, as expected. Figure 7 shows that there is an excellent agreement between POST2/CFE and AUTOLEV results. Similarly, the velocity components for the mass center of body $B$ in Fig. 8 also indicate an excellent agreement between POST2/CFE and AUTOLEV.

The angular velocity time history comparisons for bodies $A$ and $B$ are shown in Figs. 9 and 10, respectively. Again, there is excellent agreement between the two approaches. The change in the angular velocity of body $B$ at the point of release is more noticeable than for body $A$ because $B$ is smaller and has a mass distribution that differs more from that of the composite body before joint release. The main results of the CFE method are the constraint forces and moments, which are shown in Figs. 11 and 12, and are applied as additional external loads within POST2. The figures show that the forces and torques determined by CFE agree very well with the AUTOLEV results.

An important metric to assess the POST2/CFE methodology is the relative joint displacement between the two bodies when they are supposed to stay connected. This parameter is computed as the position vector from $\bar{A}$ to $\bar{B}$ and should be zero while the joint constraint is imposed for the first $10 \mathrm{sec}$. Hence, any deviation from zero is a measure

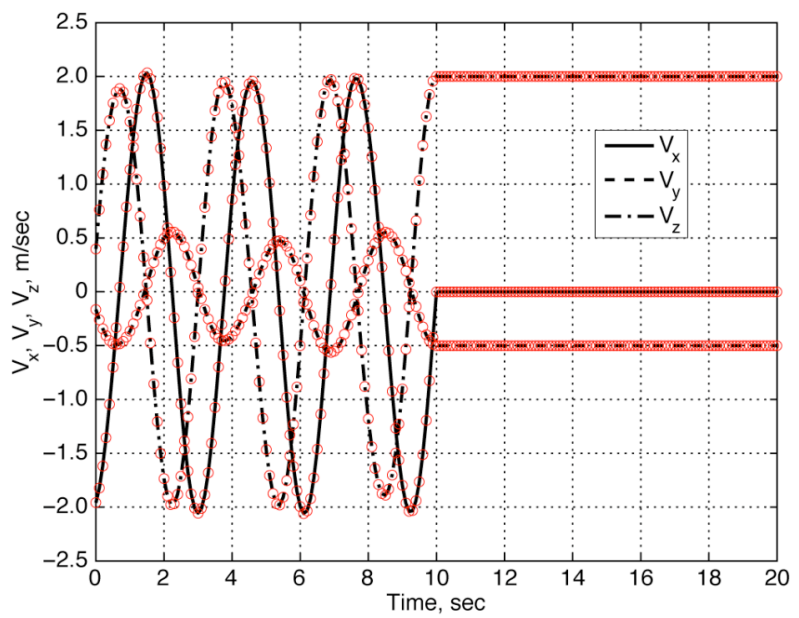

Figure 7. Test Case 1, Body $A$ : Velocity components of mass center. Joint release occurs at $t=10$ sec. POST2/CFE as circles, AUTOLEV as lines.

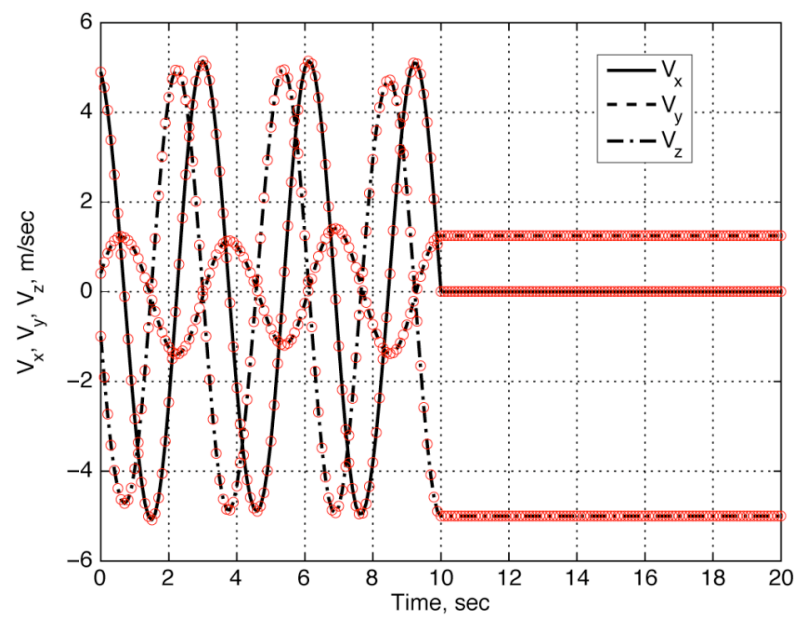

Figure 8. Test Case 1, Body $B$ : Velocity components of mass center. Joint release occurs at $t=10$ sec. POST2/CFE as circles, AUTOLEV as lines. 


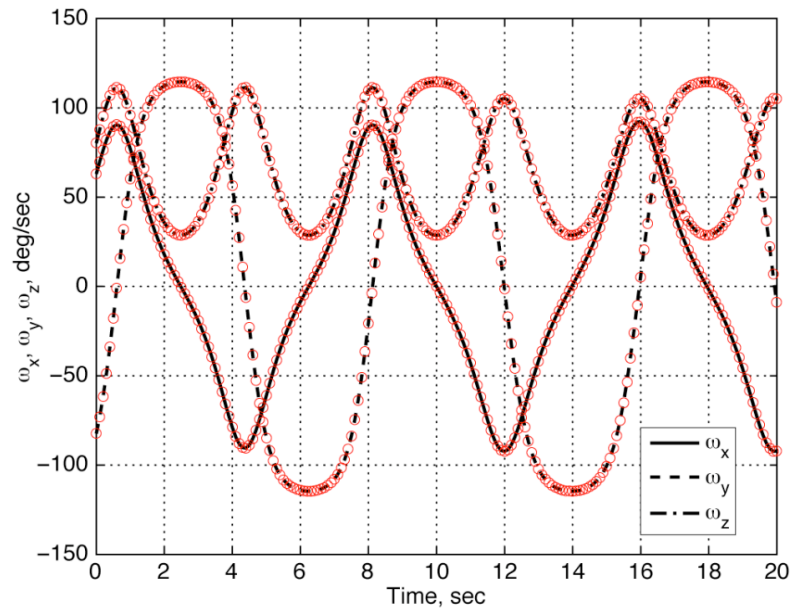

Figure 9. Test Case 1, Body $A$ : Angular velocity components. Joint release occurs at $t=10$ sec. POST2/CFE as circles, AUTOLEV as lines.

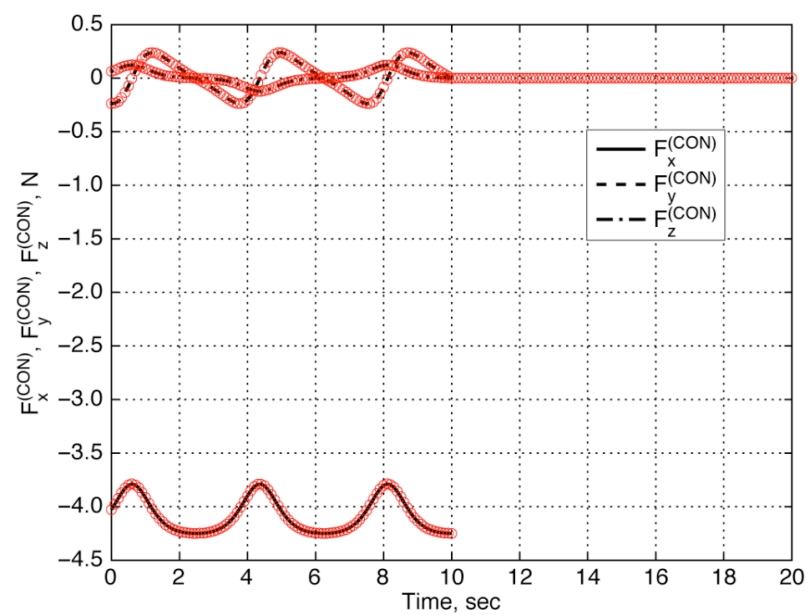

Figure 11. Test Case 1: Comparison of constraint forces required to hold bodies together. Joint release occurs at $t=10$ sec. POST2/CFE as circles, AUTOLEV as lines.

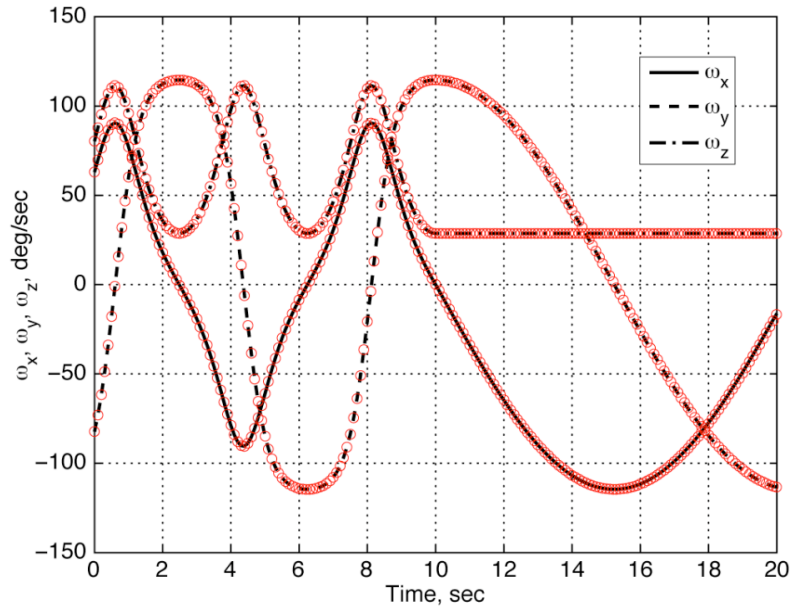

Figure 10. Test Case 1, Body B: Angular velocity components. Joint release occurs at $t=10$ sec. POST2/CFE as circles, AUTOLEV as lines.

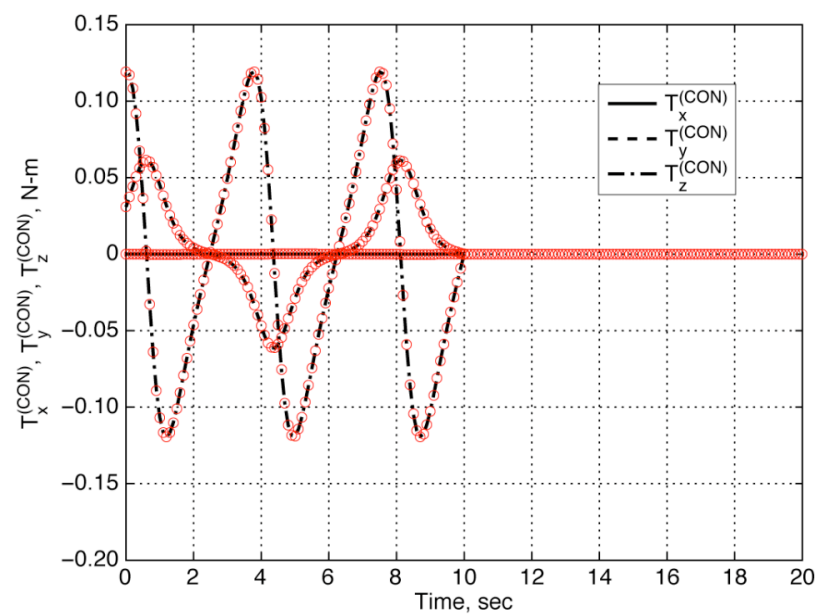

Figure 12. Test Case 1: Comparison of constraint torques required to hold bodies together. Joint release occurs at $t=10$ sec. POST2/CFE as circles, AUTOLEV as lines.

of accuracy of the CFE algorithm. In the CFE algorithm the constraints are satisfied identically at the acceleration level [Eqs. (12) and (14)]; however, as with all algorithms of this type, the constraints at the velocity and position levels are inevitably subject to numerical integration errors. Such errors are functions of the step size in a fixed-step integration scheme or the error limits in a variable-step approach. Baumgarte stabilization ${ }^{24}$ is used to control these errors from growing arbitrarily large during a simulation.

The choice of step size and Baumgarte factor for this problem represents a reasonable balance between CPU time and error buildup. Figure 13 shows the joint translational displacement as a function of time for the 10 sec period when the bodies are supposed to stay connected. In this paper, the quantities $\Delta x, \Delta y$, and $\Delta \mathrm{z}$ are the $\mathrm{x}, \mathrm{y}$ and $\mathrm{z}$ components of joint translational displacement in the local body frame and are defined as follows:

$$
\Delta \mathrm{x}=\left(\mathbf{r}_{B}-\mathbf{r}_{A}\right) \cdot \hat{\mathbf{a}}_{1}=0, \quad \Delta \mathrm{y}=\left(\mathbf{r}_{B}-\mathbf{r}_{A}\right) \cdot \hat{\mathbf{a}}_{2}=0, \quad \Delta \mathrm{z}=\left(\mathbf{r}_{B}-\mathbf{r}_{A}\right) \cdot \hat{\mathbf{a}}_{3}=0
$$

In the interest of clarity, only the POST2/CFE results are shown. The magnitude of this displacement distance is below $0.01 \%$ of the total body length. 


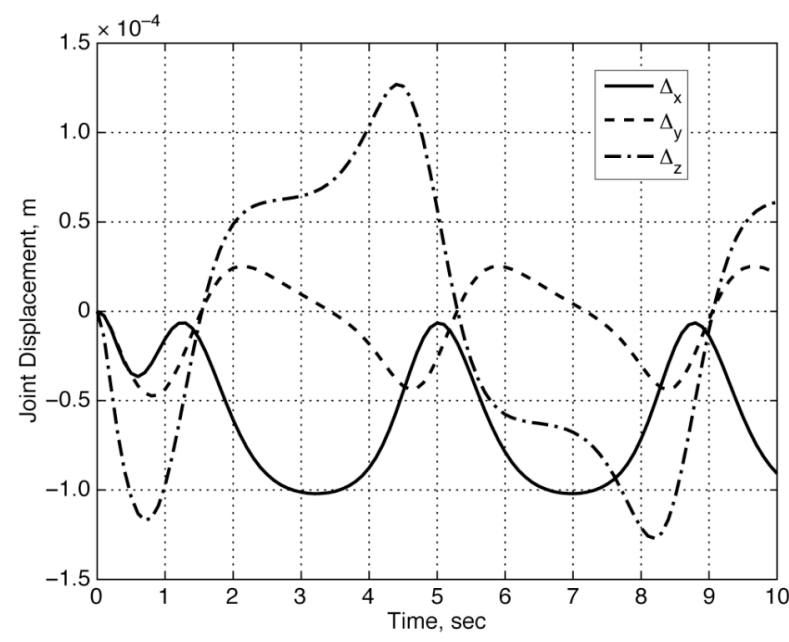

Figure 13. Test Case 1: Joint displacement in $x, y$ and $z$ directions for POST2/CFE method during time when bodies are connected. Joint release occurs at $\mathrm{t}=\mathbf{1 0}$ sec.

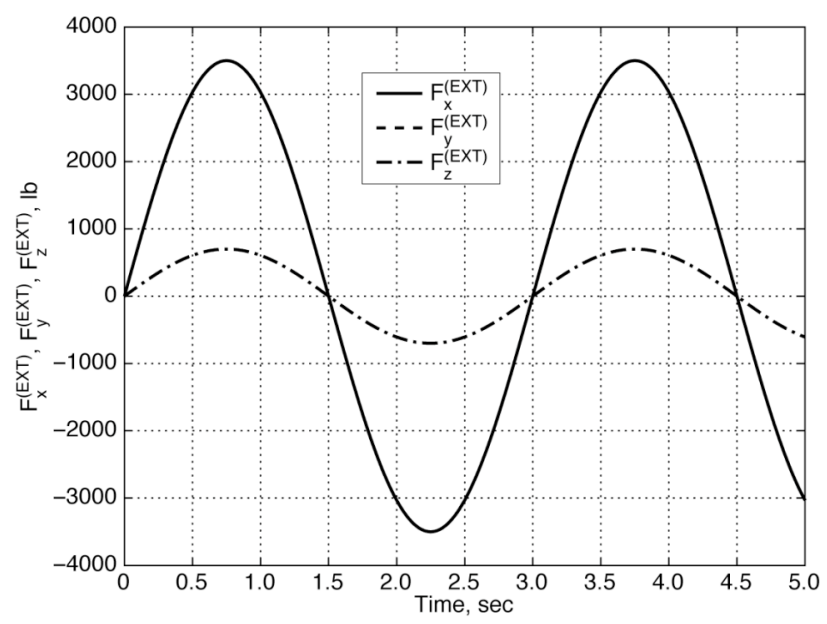

Figure 14. Test Case 2: External force components applied to Body $A$ (x and y components are identical).

\section{B. Test Case 2: Universal Joint}

To check and verify POST2/CFE results, an independent simulation using ADAMS ${ }^{12}$ software was generated. At $\mathrm{t}=0$, each body was assumed to be at rest. A time-varying force is applied to Body $A$ at a location relative to the center of mass given by the vector $-2.0 \hat{\mathbf{a}}_{1}+0.5 \hat{\mathbf{a}}_{3}$. The components of this time-varying force are shown in Fig. 14 . The mass properties of each vehicle are summarized in Table 3. The products of inertia are zero for each body in this problem.

The motion of bodies $A$ and $B$ was simulated for a duration of $5 \mathrm{sec}$. The POST2/CFE used a fixed step size integrator with step size of $0.0001 \mathrm{sec}$ with a Baumgarte factor of 5.0. The results of the simulation cases are shown in Figs. 15-18. The angular velocity of body $A$ is shown in Fig. 15. The constraint force and moment components are shown in Figs. 16 and 17, respectively. There is excellent agreement between POST2/CFE and the ADAMS solutions as evidenced by these figures. Finally, the relative joint translational displacement time histories are shown in Fig. 18. As in the previous test case of the fixed joint problem, the joint displacement is relatively small.

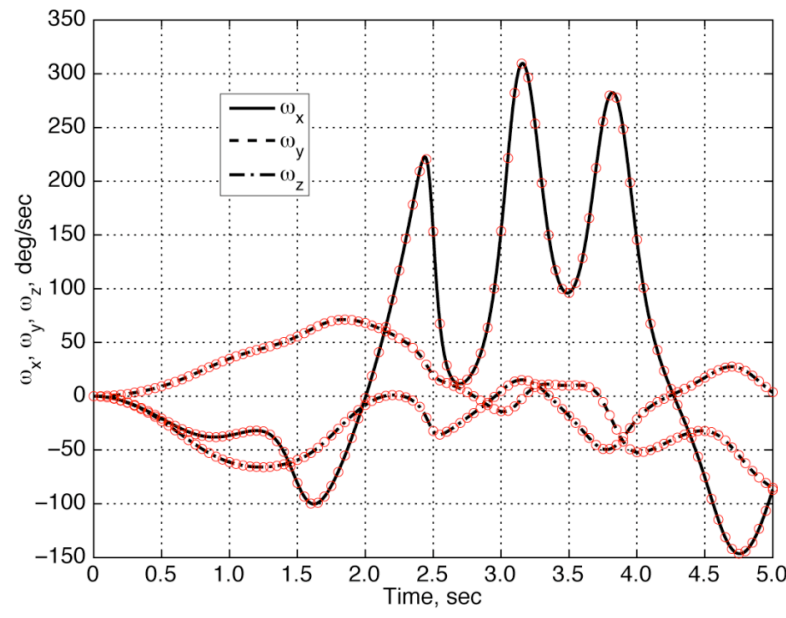

Figure 15. Test Case 2, Body B: Angular Velocity Components. POST2/CFE as circles, ADAMS as lines.

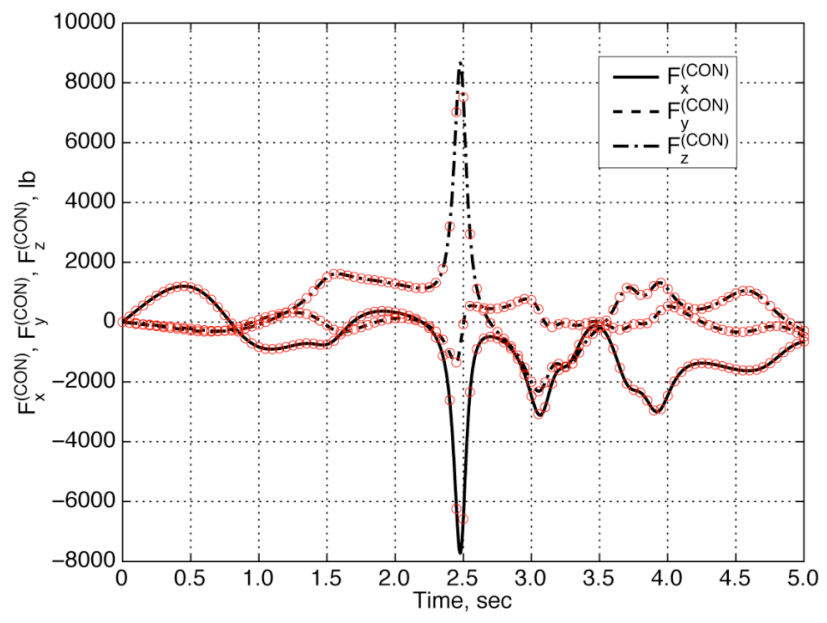

Figure 16. Test Case 2, Body $B$ : Comparison of constraint force. POST2/CFE as circles, ADAMS as lines. 


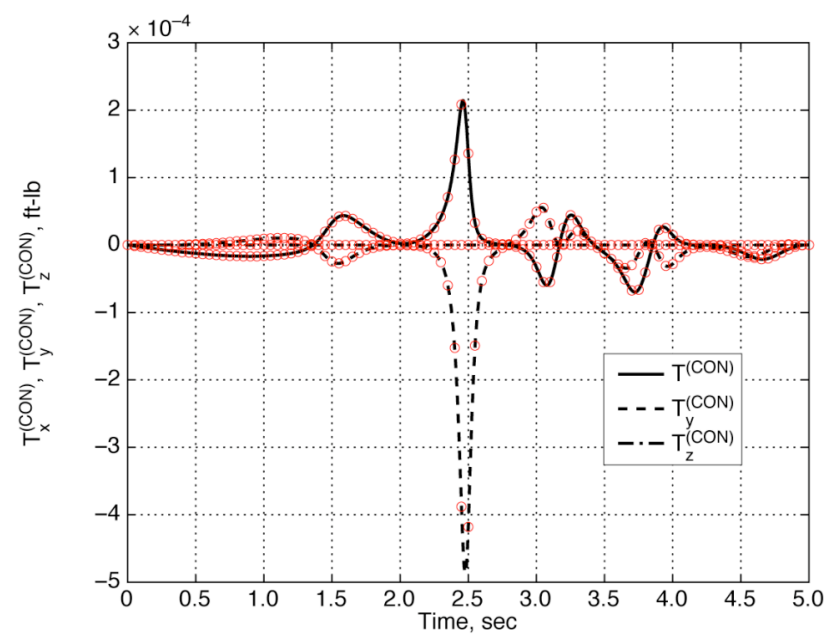

Figure 17. Test Case 2, Body B: Comparison of constraint torque. POST2/CFE as circles, ADAMS as lines.

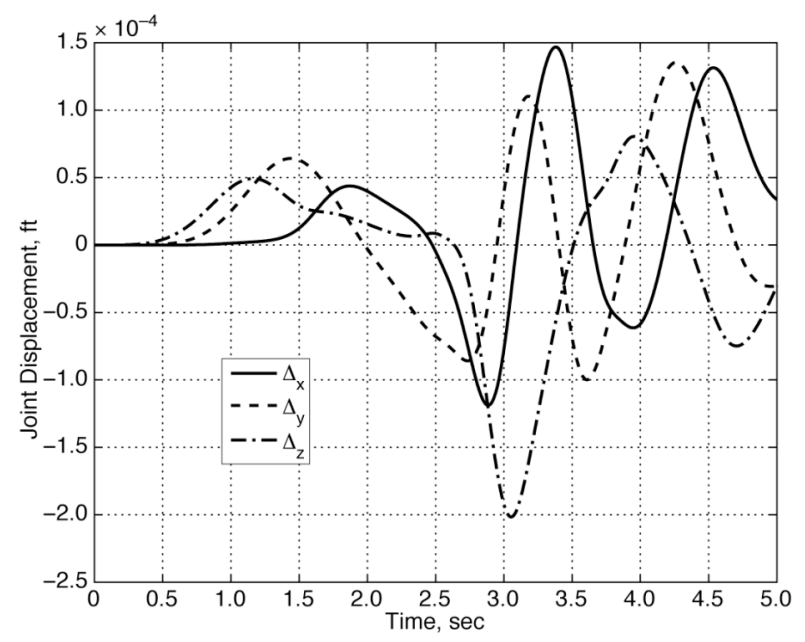

Figure 18. Test Case 2: Joint displacement in $x, y$ and $z$ directions for POST2/CFE method during time when bodies are connected.

\section{Test Case 3: Hyper X}

The results of POST2/CFE simulations were compared to previous POST2 simulations based on an engineering joint model using springs ${ }^{25}$ and the flight data. ${ }^{26}$ The engineering model employed massless springs to model sliding joints at both piston locations. As a measure of how well the joint constraint was satisfied, the relative joint displacement between the two separating vehicles is shown in Fig. 19. This parameter is the distance from the RV joint location to the HXLV joint location, and was computed in the piston axial (line-of-action, $\hat{\mathbf{a}}_{1}$ ), lateral ( $\hat{\mathbf{a}}_{2}$ ), and normal ( $\hat{\mathbf{a}}_{3}$ ) directions. The results indicate that CFE does a better job of enforcing the translational constraint, and there is virtually no joint displacement in the lateral or normal directions. Similar results are seen in Fig. 20 which shows the relative difference in attitude between the two vehicles. Again, CFE does an excellent job of enforcing the rotational constraint, essentially eliminating any relative roll and yaw between the RV and HXLV. The constraint forces and moments about the vehicle center-of-gravity that were computed by each technique are shown in Figs. 21 and 22, respectively. In these plots, the forces and moments computed by the spring model include the contributions of both pistons. The results show that both the spring model and POST2/CFE compute very similar forces and moments. However, the spring model results are oscillatory in nature since the joint constraint is not satisfied exactly.
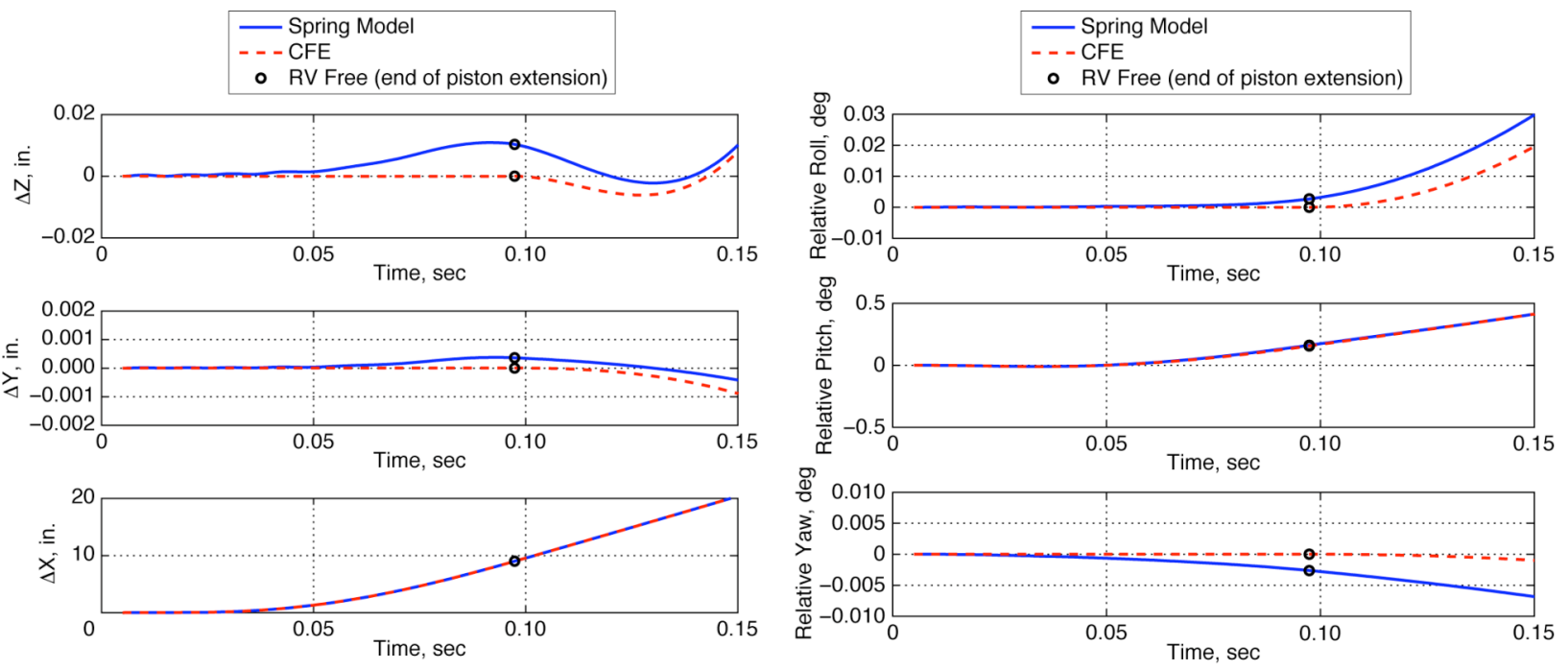

Figure 19. Test Case 3: Comparison of joint displacement in $x, y$ and $z$ directions CFE and Spring Model.

Figure 20. Test Case 3: Comparison of relative angular displacement between POST2/CFE and Spring Model. 

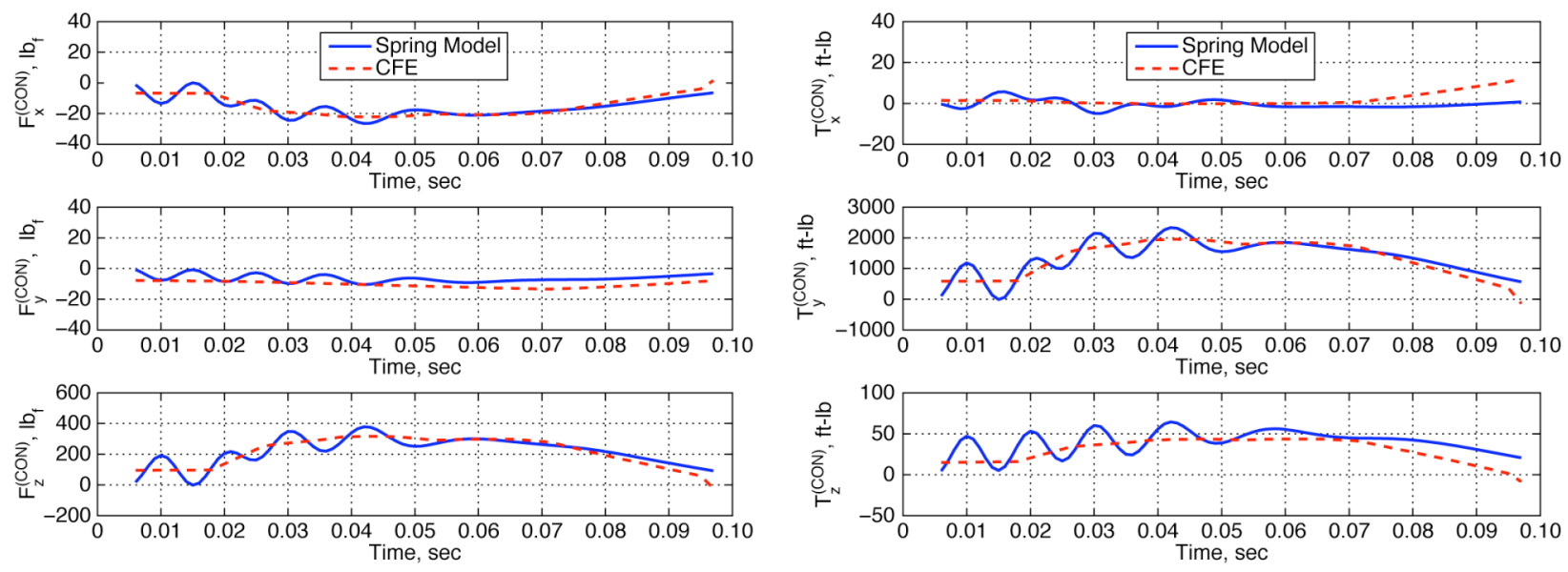

Figure 21. Test Case 3: Comparison of constraint forces computed by POST2/CFE and Spring Model.

Figure 22. Test Case 3: Comparison of constraint moments computed by POST2/CFE and Spring Model.

Comparisons of the angle-of-attack and angle-ofsideslip profiles are shown in Fig. 23. Both the spring model and POST2/CFE results match very well and are nearly identical in the angle-of-attack response. There are slightly larger differences in the sideslip response, but most of the motion occurs in the pitch plane. In addition, both simulation techniques match the observed flight data well. It should be noted that the degree to which the simulation results match the flight data depends on other factors besides CFE modeling, such as aerodynamic force and moments, winds, flight data instrumentation, etc. Aerodynamic modeling was especially critical because separation occurred at a dynamic pressure of $1000 \mathrm{psf}$. Thus, this problem is much more complex than the first two test problems where the external forces and moments were known exactly.

To illustrate the sensitivity of the simulation results to aerodynamic modeling, results are included using the nominal (pre-flight) aerodynamic database and a post-flight reconstructed aerodynamic database in which the uncertainty terms had been adjusted to better match the flight
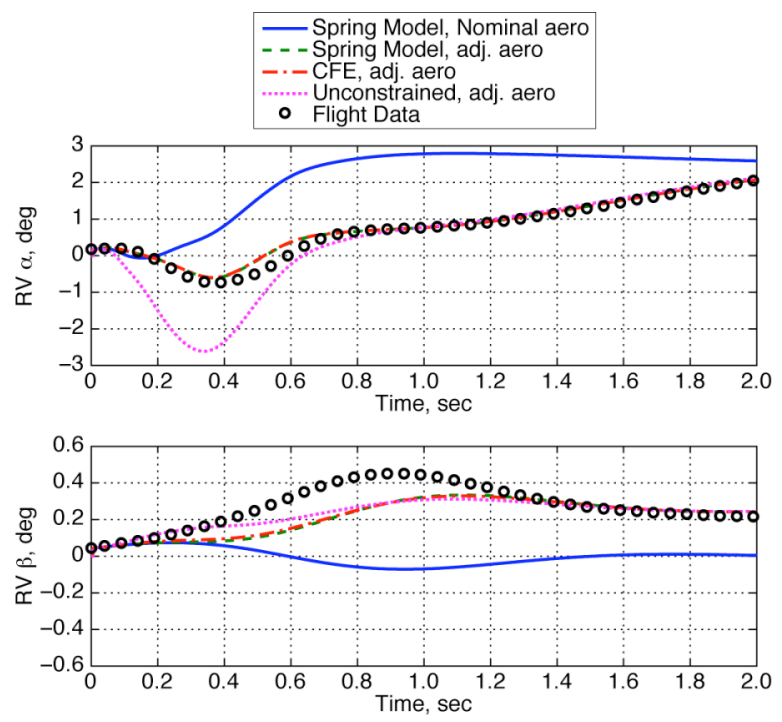

Figure 23. Test Case 3: Simulation vs flight data comparison of angle-of-attack and angle-of-sideslip profiles. data. The variations in angle-of-attack and sideslip differ significantly depending on which aerodynamic model was used. Note that all POST2/CFE results were generated using the adjusted aerodynamics.

As a further comparison, results are also shown for an unconstrained case where no constraint forces were applied to model the joint (but the piston axial force that pushed the vehicles apart was still modeled). During the first second of separation, the unconstrained results differed significantly from the cases that included the constraint, demonstrating the importance of accurate modeling of the piston joint. Even though the differences between the constrained and unconstrained angle-of-attack and sideslip profiles disappeared after the control system became fully active and was able to compensate, it is important to capture the dynamics during the first second of flight since that was when the risk of re-contact was highest.

Similar comparisons are shown for the linear accelerations in Fig. 24. The vertical (Body Z direction) acceleration results were the most sensitive to the way in which the piston constraint was modeled, and was also the most important for assessing the risk of re-contact. Again, the spring model and CFE results were nearly identical and provided a good match to the flight data. The differences between the constrained and unconstrained cases were actually larger than the differences due to aerodynamics. 

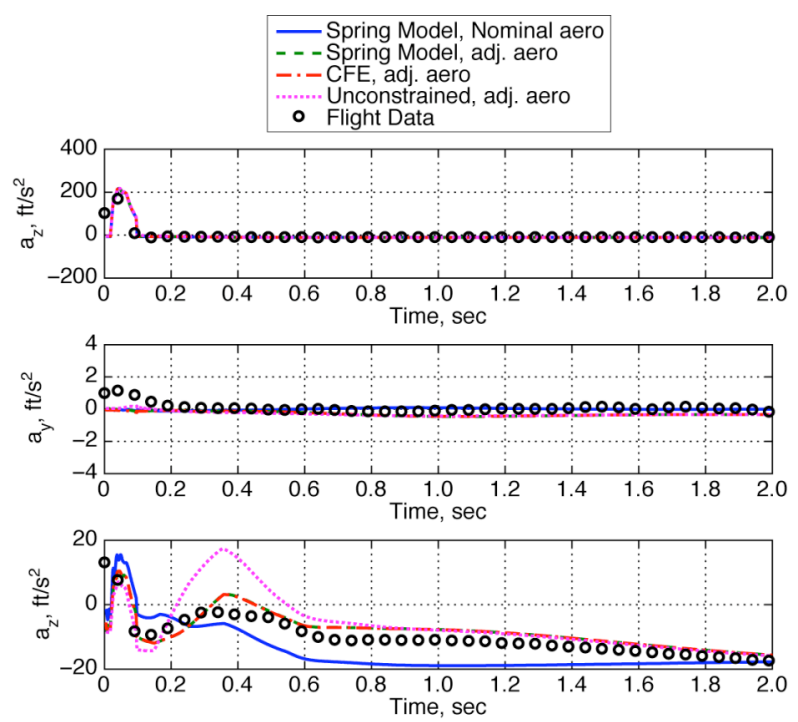

Figure 24. Test Case 3: Simulation vs flight data comparison of $x, y$ and $z$ accelerations in the local body frame.
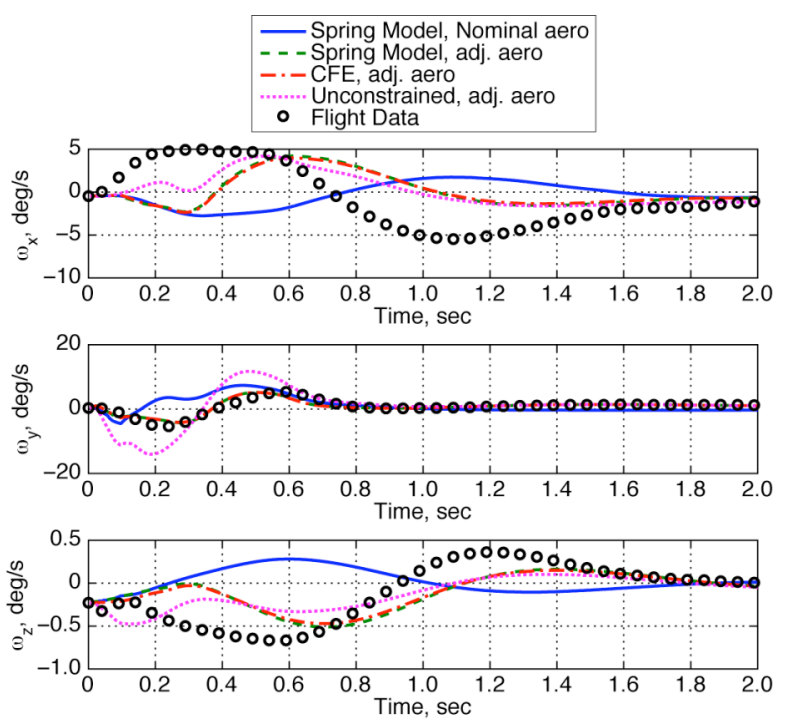

Figure 25. Test Case 3: Simulation vs flight data comparison of body roll, pitch and yaw rate profiles.

Figure 25 compares the angular velocity results with the flight data. Once again there is excellent agreement between the spring model and CFE simulation results. Both simulations match the pitch rate flight data very well and do a much better job at predicting the pitching motion than the unconstrained case. The comparison between simulation and flight for yaw rate and roll rate is not as good. Much of the difference can be attributed to inadequate lateral-directional aerodynamic modeling as is evident in the large difference in results between the cases with nominal aerodynamics and adjusted aerodynamics. Again, capturing the pitch plane dynamics was important for assessing the risk of re-contact.

\section{Concluding Remarks}

A suite of test cases was designed to verify the POST2/CFE methodology and to assess its suitability to simulate multibody separation problems in the launch vehicle staging environment. To illustrate the capability of the CFE algorithm that was implemented in POST2, three test cases of increasing complexity were presented. The first case examined the motion of a two unperturbed rigid bodies connected by a fixed joint subject to an initial rotation. The simple nature of this problem made it possible to compare simulation results to analytical predictions based on first principles. In addition, simulating a fixed joint presented a challenging case in which to assess the relative displacement between the two bodies at the joint location, a key performance metric for the CFE algorithm. The second case involved rigid bodies connected by a universal joint and typified the testing that was performed on a number of different joints applicable to stage separation problems. For the first test case, the POST2/CFE results were in excellent agreement with results obtained using AUTOLEV and for the second case, with those obtained using ADAMS.

In the third test case, the ability of POST2/CFE to model a realistic launch vehicle stage separation problem was demonstrated by simulating the X43-A (Hyper-X) stage separation problem. The POST2/CFE results agreed well with previously published POST2 results that were based on an engineering model and actually performed better at satisfying the joint constraint. Moreover, the POST2/CFE results agreed reasonably well with the flight data, and it was shown that the level of agreement degraded significantly if the constraint was not modeled.

The success of these test cases provides confidence in the ability of the CFE methodology to solve constrained motion problems in conventional trajectory simulation programs. The CFE implementation in POST2 provides the capability of simulating generic end-to-end simulations of launch vehicle trajectories.

\section{Acknowledgments}

The authors would like to gratefully acknowledge Anne Rhodes for her invaluable assistance in preparing this paper for publication, and Dr. Jim Beaty for his thorough review of the entire manuscript. 


\section{References}

${ }^{1}$ Dillenius, M. F. E., Perkins, S. C., and Nixon, D., "Pylon Carriage and Separation of Stores," Tactical Missile Aerodynamics-General Topics, edited by M. J. Hemsch, Progress in Astronautics and Aeronautics, AIAA, Washington D.C., 1992.

${ }^{2}$ Taylor, R. T., and Alford, W. J., Jr., "A Wind Tunnel Investigation of the Carry Loads and Mutual Interference Effects of 1/40-Scale Models of the X-15 and B-52 Airplanes In Combination," NASA TM X-184, 1959.

${ }^{3}$ Decker, J. P., and Wilhite, A. W., "Technology and Methodology of Separating Two Similar Size Aerospace Vehicles Within the Atmosphere," AIAA Paper 1975-29, Jan. 1975.

${ }^{4}$ Decker, J. P., Experimental Aerodynamics and Analysis of the Stage Separation of Reusable Launch Vehicles, SP-148, NASA, 1967.

${ }^{5}$ Decker, J. P., and Gera, J., “An Exploratory Study of Parallel-Stage Separation of Reusable Launch Vehicles,” NASA TN D-4765, 1968.

${ }^{6}$ Decker, J. P., “Aerodynamic Interference Effects Caused by Parallel-Staged Simple Aerodynamic Configuration at Mach Numbers of 3 and 6,"NASA TN D-5379, 1969.

${ }^{7}$ Wilhite, A. W., "Analysis of Separation of the Space Shuttle Orbiter from a Large Transport Airplane,” NASA TM X-3492, 1977.

${ }^{8}$ Naftel, J. C., and Wilhite, A. W., “Analysis of Separation of a Two-Stage Winged Launch Vehicle,” AIAA Paper 86-0195, Jan. 1986.

${ }^{9}$ Naftel, J. C., and Powell, R. W., "Aerodynamic Separation and Glideback of a Mach 3 Staged Orbiter," AIAA Paper 90-0223, Jan. 1990.

${ }^{10}$ Naftel, J. C., and Powell, R. W., "Analysis of the Staging Maneuver and Booster Glideback Guidance for a Two-Staged, Winged, Fully Reusable Launch Vehicle,” NASA TP-3335, 1993.

${ }^{11}$ Murphy, K. J., Buning, P. G., Pamadi, B. N., Scallion, W. I., and Jones, K. M., "Status of Stage Separation Tool Development for Next Generation Launch Vehicle Technologies," AIAA Paper 2004-2595.

${ }^{12}$ Using ADAMS/Solver, Mechanical Dynamics, Inc., 1999.

${ }^{13}$ Pamadi, B. N., Neirynck, T. A., Covell, P.F., Hotchko, N. J., and Bose, D. M., "Simulation and Analyses of Staging Maneuvers of Next Generation Reusable Launch Vehicles," AIAA Paper 2004-5185.

${ }^{14}$ Pamadi, B. N., Neirynck, T. A., Hotchko, N. J., Scallion W. I., Murphy, K. J., and Covell, P. F., "Simulation and Analyses of Stage Separation of Two-Stage Reusable Launch Vehicles," Journal of Spacecraft and Rockets, Vol. 44, No. 1, 2007, pp 66-80.

${ }^{15}$ Pamadi, B. N., Hotchko, N. J., Jamshid Samareh, Covell, P. F., and Tartabini, P. V., "Simulation and Analyses of MultiBody Separation in Launch Vehicle Staging Environment," AIAA Paper 2006-8033.

${ }^{16}$ Toniolo, M.D., Tartabini, P.V., Pamadi, B.N. and Hotchko, N., "Constraint Force Equation Methodology for Modeling Multi-Body Stage Separation Dynamics," AIAA Paper 2008-219.

${ }^{17}$ Brauer, G. L., Cornick, D. E., and Stevenson, R., "Capabilities and Applications of the Program to Optimize Simulated Trajectories (POST)," NASA CR-2770, 1977.

${ }^{18}$ Powell, R. W., et. al., "Program to Optimize Simulated Trajectories (POST2) Utilization Manual, Version 1.1.7," July 2002.

${ }^{19}$ McClinton, C. R., "X-43 Scramjet Power Breaks the Hypersonic Barrier, Dryden Lectureship in Research for 2006,” AIAA Paper 2006-1.

${ }^{20}$ Schaechter, D. B., and Levinson, D. A., "Interactive Computerized Symbolic Dynamics for the Dynamicist," The Journal of the Astronautical Sciences, Vol. 36, No. 4, October-December 1988, pp. 365-388.

${ }^{21}$ Kane, T. R., and Levinson, D. A., AUTOLEV 4 User's Manual, OnLine Dynamics, Inc., Sunnyvale, CA, 2005.

${ }^{22}$ Kane, T. R., and Levinson, D. A., Dynamics Online: Theory and Implementation With AUTOLEV, OnLine Dynamics, Inc., Sunnyvale, CA, 2000.

${ }^{23}$ Kane, T. R., and Levinson, D. A., Dynamics: Theory and Applications, McGraw-Hill, New York, 1985.

${ }^{24}$ Baumgarte, J., "Stabilization of Constraints and Integrals of Motion in Dynamical Systems," Computer Methods in Applied Mechanics and Engineering, Vol. 1, 1972, pp. 1-16.

${ }^{25}$ Tartabini, P. V., Bose, D. M., McMinn, J. D., Martin, J. G., and Strovers, B. K., "Hyper-X Stage Separation Trajectory Validation Studies", AIAA Paper 2003-5819, August 2003.

${ }^{26}$ Karlgaard, C. D., Tartabini, P. V., Blanchard, R. C., Kirsch, M., and Toniolo, M. D., "Hyper-X Post-Flight Trajectory Reconstruction," Journal of Spacecraft and Rockets, Vol. 43, No. 1, 2006, pp. 105-115. 